\title{
Detection of schizophrenia: A machine learning algorithm for potential early detection and prevention based on event-related potentials
}

\author{
Janek Frick \\ Aalen University, Germany \\ janek.frick@hs-aalen.de
}

\author{
Thilo Rieg \\ Aalen University, Germany \\ thilo.rieg@hs-aalen.de
}

\author{
Ricardo Buettner \\ Aalen University, Germany \\ ricardo.buettner@hs-aalen.de
}

\begin{abstract}
We show that event-related potentials can be used to detect schizophrenia with a high degree of precision. With our machine learning algorithm we achieve a balanced accuracy of $96.4 \%$, which exceeds all results with comparable approaches. For this we use additional sensors on the left and right hemisphere in addition to the common central sensors. The experimental design when recording the data takes into account the dysfunction of the schizophrenic efference copy. Due to its serious consequences, schizophrenia is a social issue in which early detection and prevention plays a central role. In the future, machine learning could be used to support early interventions. When the first symptoms appear, potential patients could be tested for the dysfunction typical for schizophrenia. In this way, risk groups and potential patients could be adequately treated before the onset of psychosis.
\end{abstract}

\section{Introduction}

Schizophrenia is a chronic psychiatric disorder characterized by the heterogeneous appearance of the symptoms hallucination, delusion and disorganization. Worldwide, slightly less than $1 \%$ of people suffer from the disease [1]. Schizophrenia patients show a significantly increased suicide rate and a shortened life expectancy of about 20 years [2]. In addition to the strong personal suffering, schizophrenia causes annual expenses of almost 94 billion $€$ in Europe [3]. The serious consequences and costs of the disease show that systematic early detection and prevention of the disease is urgently needed [4]. Such early detection enables the development, testing and implementation of intervention strategies. These can possibly prevent the outbreak of a psychosis.

Currently a diagnosis is being made using ICD-10 and DSM-5. For this purpose, the symptoms of the disease are observed and questioned through clinical interviews. This process only works if psychotic symptoms already occur [5]. Furthermore, the process is very lengthy and takes between one and six months. This is why procedures are needed to detect a possible disease at an early stage.

The first symptoms of the disease appear in childhood and adolescence, while psychotic phases usually occur much later [1]. Thus, a decline in cognitive and social skills was observed in affected adolescents [6]. Affected children sometimes also have difficulties with motor coordination [7]. Since the development of children is not homogeneous, it is not possible to draw any firm conclusions about schizophrenia based on these abnormalities alone. Therefore, biomarkers are needed that are already present at the beginning of the disease. Possible biomarkers are found in event-related potentials (ERP). An increasing number of works that recognize schizophrenia on the basis of abnormalities in ERP through machine learning have been published in the last years [8-11]. ERP is time-locked EEG activity that helps to capture neural activity related to both sensory and cognitive processes [12]. The potentials are very small voltages that are generated in the brain structure and are related to a specific event or stimulus [13]. Thus, psychophysiological correlates of mental processes can be investigated, which would not be visible in normal EEG. For the acquisition, study participants are exposed to $n$ repeating sequences of a stimulus. The brain activity measured by the EEG during the sequences is averaged over all $n$ sequences. The resulting signal reflects the electrical brain activity during the processing of this explicit stimulus. This shows characteristic features that can also be used to detect schizophrenia.

In previous work these characteristics have been evaluated on the basis of the conventional oddball paradigms by machine learning [8-11]. These show good results, but their accuracy is not yet sufficient for practical application. In this paper we show an approach that is not based on the common oddball paradigm but on dysfunctions of schizophrenics in efference copies. For this we use a very good data set and the N100 potential that occurs between $80 \mathrm{~ms}$ and $150 \mathrm{~ms}$ after stimulus. 
Through predictive efference copy, our brain is based on a survival mechanism $[14,15]$. As soon as a motor command is sent from the motor cotices to the muscles, a copy of this motor command, the efference copy, is also sent to the forward models. The forward models use this information about the motor command to predict the state of our body after the impending movement and the sensory consequences that the movement is likely to produce (sensory predictions) [16]. Thus the sensory consequences of each motor action of a person are predicted by an efference copy and compared after the execution of the action [14].

Efference copies have a great influence on the control of speech motor functions. Several studies have shown that a correct prediction of the acoustic consequences of one's own speech production is an integral part of the speech motor control mechanism [17]. When a tone itself is produced verbally, the efference copy prepares the auditory cortex for sensory input. Recent studies have shown that the diminished cortical response, interpreted as an indication of a functioning copy of an effect, is already produced when a tone is not produced verbally but by pressing a button followed by a tone [18]. If the task of the button-tone has already been mastered several times, the brain prepares the motor response of the button press as well as the expected acoustic input. As can be seen in Figure 1, the amplitude of the cortical signal when hearing a tone (blue) in healthy people is greater than the amplitude when processing a self-produced tone (green).

The dysfunction of efference copies in schizophrenia patients has been described in several papers $[18,19]$. It has not been used for early detection by machine learning so far. With this paper we want to clarify the question whether schizophrenia can be effectively detected by the dysfunction of efference copy using machine learning. Furthermore, we want to identify which sensors contribute significantly to the classification of schizophrenia. In addition to the centrally placed sensors, sensors of the right and left hemisphere were therefore also included in the study. In schizophrenia patients, abnormalities are found in both the left and right hemispheres of the brain. Thus, schizophrenia patients often find it difficult to correctly process semantic aspects of language, which is mainly attributed to the left hemisphere [20]. There is also evidence that auditory verbal hallucinations are caused by a perceptual disorder that originates in the left hemisphere [21]. These auditory hallucinations are a key symptom of schizophrenia. If we look at the right hemisphere of patients with schizophrenia, the right hemisphere shows reduced activity. This reduced activity is associated with the social disorders of patients. Thus, healthy people show a higher activity in the right prefrontal brain regions during emotion processing than schizophrenia patients [22].

The four most important contributions of our work are:

- We were the first to evaluate event-related potentials with underlying efference copy by a Random Forest.

- We outperformed the current benchmark with an accuracy of $88.25 \%$.

- Thanks to Random Forest, we were able to calculate the influence of individual sensors on our accuracy, which gives additional information.

- Our approach is accurate, fast and cost-effective, which contributes significantly to information systems research in healthcare [23] .

The presented approach is of practical relevance as a contribution to the search for biomarkers of schizophrenia. Early preventive treatment of risk groups supports those affected. On the other hand, it will help to reduce and better calculate the cost of the disease in the future [24]. Such new, data-driven procedures are important for the health care system $[25,26]$.

\section{Related Work}

In recent years, several interesting approaches to identifying schizophrenia using machine learning and ERP data have been published. Their results are summarised in Table 1. Neuhaus et al. published an approach based on a visually and auditory stimulated N100 potential [10]. For this purpose, ERP data of 24 schizophrenics and 24 healthy subjects were collected. The N100 and P300 potential was used to train different machine learning algorithms. Accuracy was between $53.6 \%$ and $72.4 \%$ on the test set, with the best result achieved by the KNN. In this work Neuhaus et al. argued that the accuracy could probably be increased by a larger number of subjects. In 2014 Neuhaus et al. published a study with a significantly larger data set of 288 subjects. The potentials P50, N100 and P300 were investigated. The accuracy could be increased to $77.7 \%$, whereby a single case classification was chosen [8]. Neuhaus et al. argue that a new approach to the classification could be necessary due to the poor dicriminatory properties in single subject classification.

Laton et al. published a paper in 2014 in which schizophrenia was diagnosed on the basis of ERP data [11]. They selected auditory and visual stimulation of P300 potential and mismatch negativity as the experimental design; the data were collected from a total 


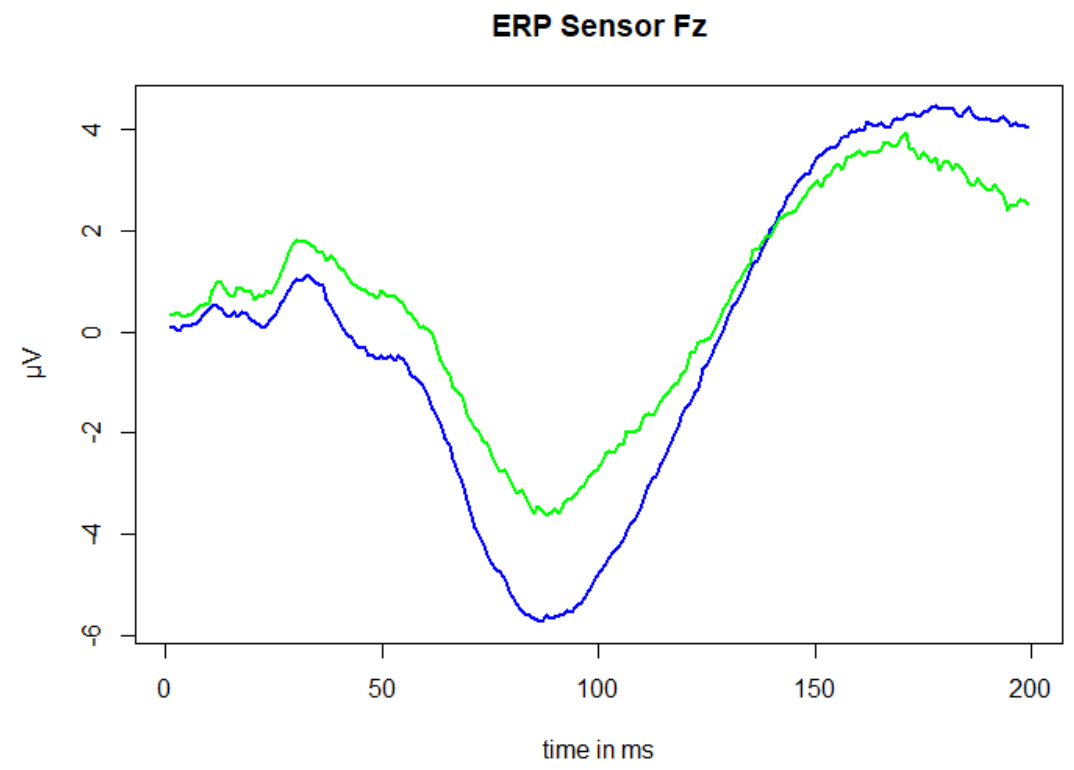

Figure 1. Event-Related Potential of healthy participants at sensor Fz after acoustic stimulus (blue) and after self-evoked acoustic stimulus (green).

of 108 subjects. Several machine learning algorithms were trained, with the best result of $85 \%$ accuracy achieved by the Random Forest classifier.

Shim et al. published an approach in 2016 in which the data of 68 subjects were also classified as either sick and healthy [9]. The auditory P300 potential was used together with the average cortical activity of the subjects to train an SVM algorithm. Together, this feature selection resulted in an accuracy of $88.25 \%$, with only the auditory P300 potential leading to an accuracy of $80.88 \%$.

Table 1. Comparable machine learning based approaches

\begin{tabular}{lccc}
\hline \multicolumn{4}{c}{ approaches } \\
\hline Author & Year & Accuracy & Reference \\
\hline Neuhaus et al. & 2012 & $72.40 \%$ & {$[10]$} \\
Neuhaus et al. & 2013 & $77.70 \%$ & {$[8]$} \\
Laton et al. & 2014 & $85.00 \%$ & {$[11]$} \\
Shim et al. & 2016 & $88.25 \%$ & {$[9]$} \\
\hline
\end{tabular}

While these papers have already delivered good results and relevant insights, there is still much space for new approaches to achieve better results.

\section{Method}

Since the goal of our work was to create an IT artifact, we followed the procedure according to Hevner et al. [27].
The complete research process used in this work is shown in Figure 2.

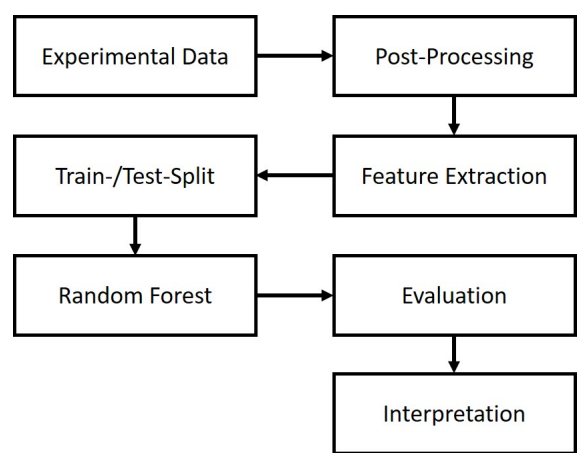

Figure 2. Flowchart of the research process.

As a first step the data was post-processed. In order to train the machine learning algorithm, features were extracted and the data set was split into a train- and test set. A Random Forest algorithm was trained, which was then evaluated and interpreted.

\subsection{Dataset and EEG noise removal}

The data set we used for this work was produced by the National Institute of Mental Health (NIMH) and is freely available on the internet under project number R01MH058262. All volunteers have given their written consent to participate. The costs of the study were borne 
by the NIMH. The results of the study were published in Schizophrenia Bulletin in 2014 [18]. One data set with a total of 58 subjects was collected. The data set was subsequently supplemented by 23 subjects with whom the identical experiment was carried out at an earlier point in time. Thus, the data set comprised 81 subjects. All subjects were recruited by advertising and word of mouth. The disease schizophrenia was diagnosed in an interview according to DSM-4. Due to the need for comparability between the two groups, the control group was selected for demographic characteristics similar to those of the diseased group. The demographic characteristics of the subjects are shown in Table 2.

Table 2. Demographics of schizophrenics (SC) and

\begin{tabular}{lcc}
\multicolumn{3}{c}{ healthy (HC) subjects } \\
\hline \multicolumn{1}{c}{ SC } & HC \\
\hline $\mathrm{n}$ & 49 & 32 \\
Men & 41 & 26 \\
Women & 8 & 6 \\
& & \\
Age ( $\varnothing$ years) & 40.02 & 38.38 \\
SD (years) & \pm 13.48 & \pm 13.92
\end{tabular}

In the NIMH experiment, the subjects pressed a button every $1-2$ seconds to produce a $1000 \mathrm{~Hz}$ and 80 $\mathrm{dB}$ tone without delay. This was done for 100 repetitions. The generated tones were then played back. In a final experiment, the button was pressed in the same rhythm as before without playing a sound. While these tasks were being performed, the brain activities of the subjects were recorded by a BioSemi ActiveTwo EEG system. For this purpose, 64 electrodes were attached to the scalp, which recorded voltage changes at $1024 \mathrm{~Hz}$. The electrodes were attached to the head according to the 10-20 standard layout [28].

A crucial problem with EEG data sets is the strong noise generated during data collection. The electrodes attached to the scalp must record the finest signals from the brain. This also leads to mixed signals being recorded. In particular, the movement of the eyes, blinking, muscle activity and heartbeat are found as disturbance data in the EEG data set. The collected data are therefore post-processed after recording.

Since EEG data are electrical potential fluctuations, a reference electrode with constant equilibrium potential is required. This must be placed in a neutral location such as the nose, chin or earlobes. No electrical brain activity is measured at these locations. In the case of the data set collected by NIMH, the two electrodes were attached to the earlobes of the test persons. In a first step, the data of the 64 electrodes were referenced to the averaged reference electrodes. To post-process data, a $0.5 \mathrm{~Hz}-15 \mathrm{~Hz}$ bandpass filter was applied [18]. This reduces low-frequency interference signals. These can be found, for example, in sweat artifacts. The post-processing of the data thus consisted of the referencing of the signals to the reference electrode and the signal filtering by the band-pass filter from $0.5-15 \mathrm{~Hz}$. The data were then broken down into 3-second periods, so that each period contained a stimulus. 1.5 seconds before and 1.5 seconds after experimental activity were recorded. A baseline correction was then performed. After baseline correction, further interfering artifacts were removed. The muscle movements of the volunteers, such as blinking or eye movements, cause interfering signals in EEG data. In order to filter these signals from the data, electrodes were attached to the corners of the eyes of the volunteers. In addition, two electrodes were placed above and below the right eye. These were used to record electrooculography (EOG). There is an electrical potential between the cornea and the fundus of the eye: the cornea is positively charged, the posterior part of the eye negatively [29]. The electrical potential of the eye radiates into surrounding tissue. Eye movements bring the positively charged cornea closer to one of the electrodes so that a field change can be measured [29]. Using a regression algorithm, the noise caused by blinking and eye movements was filtered from the EEG data using the EOG data [18].

Event-related potentials were then extracted by averaging procedures. The ERPs are not visible in the EEG because their amplitude of $\langle 30 \mu \mathrm{V}$ is superimposed by the spontaneous activity of the EEG. In order to make the signal visible, the test subject is repeatedly exposed to a stimulus. Afterwards the signal is averaged. This is based on the assumption that event-related potentials always occur at a fixed time. A repetition of the action leads to an identical course, while the spontaneous activities that accompany them fluctuate randomly from period to period. This spontaneous activity cancels each other out, while the desired signal becomes visible. Figure 1 shows both the raw EEG sequences at sensor $\mathrm{Fz}$ and the resulting ERP signal.

\subsection{Machine Learning Method}

As outlined above, the aim of this paper was to provide a robust and well-classifying model that could be used in medical schizophrenia diagnosis. The step-by-step process of the experiments is shown in Figure 3, the whole pre-processing process in Figure 5.

Event-related potentials consist of the four components: amplitude, polarity, latency and topography. 


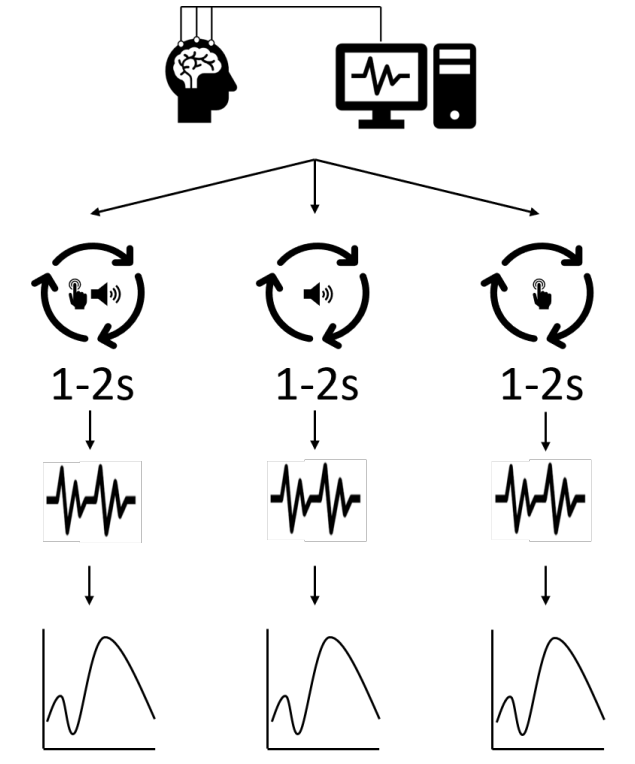

Figure 3. Three experiments were conducted. The collected EEG data was then processed to ERPs.

The data set for the creation of a classification model was created taking these components into account. As shown in Figure 3, the original data set consists of data from three experiments: (i) a button is pressed every 1-2 seconds to produce a sound (ii) the sounds are played back without pressing a button, (iii) the button is pressed every 1-2 seconds without playing a sound.

Since only the stimulus processing of the brain is to be considered, the measured values from (iii) were subtracted from the values from (i). The result is a data set containing the individual's perception of stimuli evoked by themselves.

In order to obtain a robust model, the individual ERPs were averaged, resulting in a dataset with "Healthy Controls" and "Schizophrenia Patients". In view of the manageable size of the data set, such a procedure is preferable to individual case classification [8]. While comparable work frequently focused on central sensors, this work should also take left and right hemispheric sensors into account. The sensors $\mathrm{Fz}, \mathrm{FCz}, \mathrm{Cz}$ as well as F3, C3, CP3 and FC4, C4 and CP4 were therefore included in the data set. Sensors on the left hemisphere are provided with odd, sensors on the right hemisphere with even numbers; central sensors contain a characteristic $\mathrm{z}$. As a further relevant characteristic the time was additionally appended to to adequately consider possible latencies in the stimulus processing.

The potential influenced by the efference copy is the N100 potential. It occurs $80-150 \mathrm{~ms}$ after stimulus and is therefore an endogenous component. Endogenous components depend on cognitive and psychological

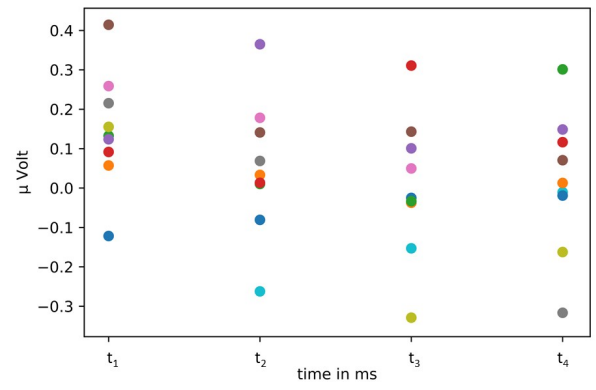

Figure 4. The signal from each sensor at each time $t_{n}$ was extracted and used as feature input to train the machine learning model.

factors that can be influenced by individual attention and the nature of the task at hand. In healthy individuals, a significantly inhibited N100 amplitude occurs when a sound is generated by pressing a button, indicating less necessary neuronal activity [30, 31]. By correctly predicting the input by the efference copy, tone processing causes less activity. Figure 1 illustrates the mechanism of efference copy. Since the event-related potential N100 is measured in the period of $80 \mathrm{~ms}-150 \mathrm{~ms}$, only this time window was considered for the investigation. All relevant characteristics of event-related potentials - amplitude, polarity, latency and topography - were measured on nine sensors and used as a data set. This results in a total data set with 144 observed sensor values at each of the nine sensors as well as the time stamp. The individual time periods resulting from the previously calculated individual perception of stimuli caused by the subject itself (Exp. (iii) minus Exp. (i)) and the N100 range were then used as features. The features for each sensor at four different points in time are shown in Figure 4.

The classification should be based on a model that is as robust as possible and allows conclusions to be drawn about the relevance of individual sensors. In order to answer the question which the most important sensors for the classification of schizophrenia are, Random Forest was chosen as the classifier. The amount of data could be efficiently processed by the Random Forest. In addition, a direct conclusion could be drawn on the most important variables for classification. The Random Forest was originally designed by Breiman [32]. It consists of a collection of tree predictors, each tree based on independently selected vectors. The classification outputs of the individual trees are used to determine the overall classification. This is based on the idea that the aggregated decisions of several trees are superior to those of a single tree. 
In a first step, the data set is divided into two parts: the training dataset and the test data set. A model is trained on the training dataset, which is then validated on the test data. The training data are transferred to the Random Forest, where they are randomised in a first step. This randomisation, known as bagging ("bootstrap aggregating") also originates from Breiman [32]. Bagging creates new randomised data sets that have the same cardinality as the original data set. The Random Forest process was defined by Liaw and Wiener as follows [33]: (i) Draw ntree bootstrap samples for the original data, (ii) for each of the bootstrap samples, grow an unpruned classification or regression tree, with the following modification: at each node, rather than choosing the best split among all predictors, randomly sample mtry of the predictors and choose the best split from among those variables, (iii) predict new data by aggregating the predictions of the ntree trees (i.e. majority votes for classification, average for regression).

As part of our classification, the Random Forest of the caret package was used. The data set was divided as follows: training $80 \%$ and testing $20 \%$. The number of trees was $n=500$. The mtry $=10$ considered all available variables: nine sensors as well as the time. The entire step by step process of data preparation is shown in Figure 5. The variable importance calculated by Random Forest was also saved.

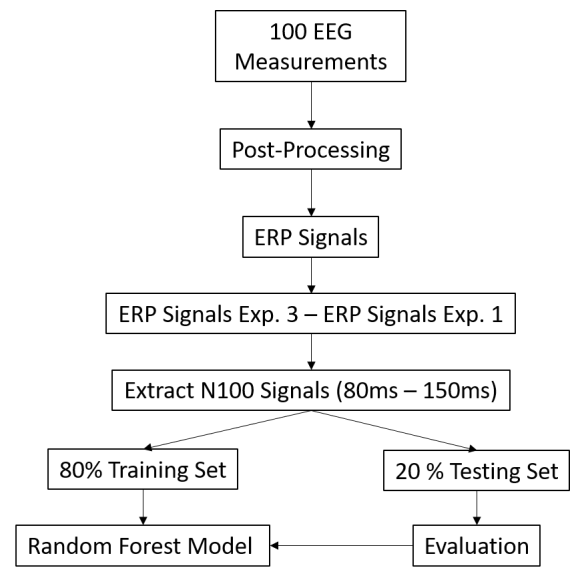

Figure 5. Flowchart of the step-by-step process from the data acquisition to the model evaluation.

In order to achieve a reliable value, $\mathrm{k}$-fold cross-validation was applied. In the cross-validation process, the data set is divided into $k$ equal parts. The training is performed on $k-1$ data, the validation on the excluded part, which is thus used as the test data set. This process is repeated $k$ times so that each part is also used as a test data set. $k$ was set to 10 for this work.

As result, we were told which sensor values were classified correctly and which were classified incorrectly on the basis of the trained model. For this purpose, the confusion matrix of the model was output. The confusion matrix classifies as follows: (i) True Positive: The sensor values belong to the data set of the healthy subjects and the model assigned them correctly. (ii) False Negative: The sensor values belong to the data set of the healthy subjects and the model incorrectly assigned them to the sick subjects. (iii) False Positive: The sensor values belong to the diseased subjects but were assigned to the healthy subjects. (iv) True Negative: The sensor values belonged to the group of sick subjects and were correctly assigned by the model.

\section{Results}

Table 3. Predictive Result of Model

\begin{tabular}{lrr}
\hline Performance Indicator & Value & SD \\
\hline Balanced Accuracy & $96.4 \%$ & $7.57 \%$ \\
Sensitivity & $92.8 \%$ & $13.55 \%$ \\
Specificity & $100.0 \%$ & $0.00 \%$ \\
Positive Predictive Values & $93.3 \%$ & $9.92 \%$ \\
Negative Predictive Values & $100.0 \%$ & $0.00 \%$ \\
Kappa & $93.0 \%$ & $13.55 \%$ \\
\hline
\end{tabular}

Of the nine sensors, 144 values were recorded for the N100 potential. The classification model was trained with $80 \%$ of the data and tested for the remaining $20 \%$. Thus, the model was tested on 28 points of the time series. The best classification model of the N100 potential achieved an accuracy of $96.4 \%$. All performance metrics can be seen in Table 3 .

Table 4. Confusion Matrix of Random Forest model using the test dataset

\begin{tabular}{|c|c|c|c|}
\hline \multirow{4}{*}{ Prediction } & \multicolumn{3}{|c|}{ Reference } \\
\cline { 2 - 4 } & & $\mathbf{0}$ & $\mathbf{1}$ \\
\cline { 2 - 4 } & $\mathbf{0}$ & $\mathbf{1 3}$ & 0 \\
\cline { 2 - 4 } & $\mathbf{1}$ & 1 & $\mathbf{1 4}$ \\
\hline
\end{tabular}

In addition to the balanced accuracy of $96.4 \%$, a sensitivity of $92.8 \%$ was achieved. The specificity even reached $100 \%$. Altogether only one point of the time series was wrongly assigned, as shown in the confusion matrix in Table 4 . The variable importance, shown in Figure 6, also provides valuable information: the most important sensor for the Random Forest is the CP4 at the right hemisphere. This is followed by the central sensor Fz followed by the left hemispheric sensor CP3. The fourth most important feature is time. 


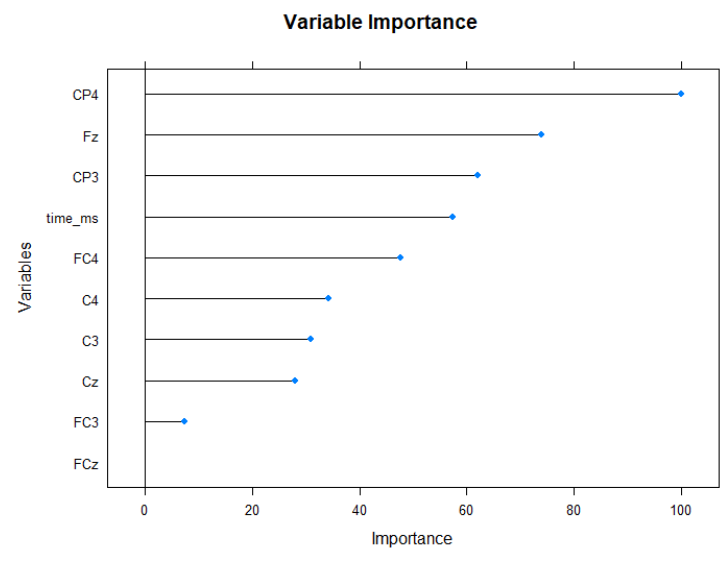

Figure 6. Variable Importance of the Features used by the Random Forest Classifier.

\section{Discussion}

While schizophrenia is currently diagnosed by observation and questioning according to ICD-10 and DSM-5, the number of publications is constantly increasing with the search for relevant biomarkers. Biomarkers are extremely relevant in this case, as diagnosis currently takes a long time and can be negatively influenced by misinformation from the patient [9]. Furthermore, biomarkers can also be used in the urgently needed early detection. In addition to genetic markers, ERP data evaluated by machine learning are increasingly being used for this purpose. Considering the technological development in the EEG segment, these approaches could be implemented cheaply, easily and quickly in the healthcare sector in the future. Thus, the first mobile EEG devices already show a good accuracy in measuring ERPs [34].

The approaches to ERP evaluation by machine learning already presented here have produced good results, but also show that new approaches are needed to detect schizophrenia even more accurately [8-11]. In contrast to the approaches listed in Table 1, we only considered the N100 potential, which in most studies is only measured at the central sensors $\mathrm{Fz}$ or Cz. We added the sensors F3, C3, CP3, and F4, C4 and CP4. Thus, further possible features are added to the Random Forest in order to achieve a good classification result. Furthermore, the influence of the left and right hemisphere of the brain on the classification result was displayed by the variable importance and the Sensors F3, $\mathrm{C} 3, \mathrm{CP} 3, \mathrm{~F} 4, \mathrm{C} 4$ and $\mathrm{CP} 4$.

The functionality of the efference copy of healthy and diseased people was investigated by the described button-tone experiment. The dysfunction of the schizophrenic efference copy reflected in N100 should have a positive effect on the classification model as the Random Forest recognizes clear classification patterns. It is currently assumed that the absence or dysfunction of the auditory efference copy is the main cause of the acoustic hallucinations of schizophrenics. Many of the patients suffer from this symptom [35-37]. The pattern recognition of the potential N100 of self-evoked stimuli has corresponding relevance. After extensive literature research and to the best of our knowledge, there is currently no scientific work available that has classified schizophrenia on the basis of the dysfunction of the auditory efference copy by Random Forest.

Looking at the achieved results, a good model could be created with an accuracy of $96.4 \%$, which surpasses other current works. In contrast to many other scientific papers, time as well as right and left hemispheric sensors were taken into account $[38,39]$. As shown by the output of the variable importance, this feature selection was crucial to achieving this very good result. The otherwise neutral time value in combination with the corresponding sensor values became very important variables for prediction. Such delayed stimulus processing has already been proven many times $[40,41]$. The relevance of the sensor Fz in connection with the N100 potential was mentioned before and could also be proven in this work. As the second most relevant variable, the sensor values at Fz had a large influence on the trained classification model. However, the most relevant result of this work is the strong influence of the left and right hemispheric sensors CP3 and CP4. Both posterior sitting sensors were extremely relevant in creating the classification model. While in many scientific studies it is primarily the central sensors that have been considered, it could be shown that the consideration of further selected sensors can have a strong positive influence on further research results. A positive characteristic of our approach is that each data point $t_{n}$ of the time series is considered separately. Thus, future ERP data of schizophrenia patients could be tested individually at each of the time points between 80 $-150 \mathrm{~ms}$.

In the context of early intervention, Sommer et al. also address the potential of ERP for early detection of the disease [4]. Detection and intervention in schizophrenia at an early age is a priority in schizophrenia research [4]. This detection could solve the problem of long diagnosis times. Drugs and therapeutic support can be provided at an early point in time. The effects of the serious illness could thus be reduced. Initial work also indicates that early intervention can reduce the costs of the disease [42]. A prevention system would therefore help both the patient and the healthcare system. A new approach to prevention could be used as a non-invasive method. With our approach we want 
to participate in the search for a non-invasive method of prevention and build on already existing approaches.

\section{Conclusion}

In this work, we present an approach that could be applied in the early detection of schizophrenia using ERP. The dysfunction of the predictive efference copy in schizophrenics was the subject of the experimental design and thus the data basis of our work. In addition, an approach was presented in which the data of the test persons were averaged according to their affiliation in healthy and sick persons, in order to subsequently transfer the ERP sensor values to the machine learning model at every time point $t_{n}$. The centrally positioned sensors, which are normally used for ERP studies, were supplemented by additional sensors of the left and right hemisphere. It was shown that these additive sensors provide important information for the algorithm. Our model outperformed comparable works with a very good level of accuracy of $96.4 \%$. As explained, such an algorithm could help in the future to detect the disease early and intervene accordingly.

\subsection{Limitations}

Since the results of our research are recent, the algorithm has not yet been tested in a clinical environment and thus in practice. Furthermore, the results have not yet been tested for external validity. No other algorithms have been trained. Also the influence of medication on the model could not yet be tested. The data set used is also not very large, which can lead to variations in the performance of the algorithm.

\subsection{Future work}

To further advance our schizophrenia diagnosis algorithm, in terms of performance and validity, one of the main objectives of our future work is to re-evaluate the algorithm using a larger data set that contains a higher number of subjects. To make further progress in the diagnosis of schizophrenia, we would like to combine another recently presented new ML approach with the approach presented here. The other approach uses EEG data that is divided into small frequency ranges and evaluated by machine learning. With this approach, different diseases addictions and traits can be detected reliably [43-56]. The combination of these two approaches could also make progress possible in the areas of stress, concentration, mindfulness and cognitive workload [57-63]. Furthermore, we will triangulate psychophysiological and physiological data (i.e., electroencephalographic data and spectra, electrocardiographic data, electrodermal activity, eye fixation, eye pupil diameter, facial data to increase reliability [64-68]. In addition, we will evaluate technology acceptance and trust in multi-agent-simulations [69-77]. Furthermore, we will apply a deep learning approach using convolutional neural networks [78-82]. With these approaches we can improve our classifier performance and further decode schizophrenia.

\section{Acknowledgements}

This research is funded by the Carl Zeiss Foundation and the German Federal Ministry of Education and Research (13FH4E03IA, 13FH4E07IA, 13FH176PX8, 13FH4E05IA).

\section{References}

[1] R. S. Kahn, I. E. Sommer, R. M. Murray, A. Meyer-Lindenberg, D. R. Weinberger, T. D. Cannon, M. O'Donovan, C. U. Correll, J. M. Kane, J. van Os, and T. R. Insel, "Schizophrenia," Nature Reviews Disease Primers, vol. 1, no. 1, pp. 1-23, 2015.

[2] T. M. Laursen, M. Nordentoft, and P. B. Mortensen, "Excess Early Mortality in Schizophrenia," Annual Review of Clinical Psychology, vol. 10, no. 1, pp. 425-448, 2014.

[3] A. Gustavsson, M. Svensson, F. Jacobi, C. Allgulander, J. Alonso, E. Beghi, R. Dodel, M. Ekman, C. Faravelli, L. Fratiglioni, B. Gannon, D. H. Jones, P. Jennum, A. Jordanova, L. Jönsson, K. Karampampa, M. Knapp, G. Kobelt, T. Kurth, R. Lieb, M. Linde, C. Ljungcrantz, A. Maercker, B. Melin, M. Moscarelli, A. Musayev, F. Norwood, M. Preisig, M. Pugliatti, J. Rehm, L. Salvador-Carulla, B. Schlehofer, R. Simon, H.-C. Steinhausen, L. J. Stovner, J.-M. Vallat, P. V. den Bergh, J. van Os, P. Vos, W. Xu, H.-U. Wittchen, B. Jönsson, and J. Olesen, "Cost of disorders of the brain in Europe 2010," European Neuropsychopharmacology, vol. 21, no. 10, pp. 718-779, 2011.

[4] I. E. Sommer, C. E. Bearden, E. van Dellen, E. J. Breetvelt, S. N. Duijff, K. Maijer, T. van Amelsvoort, L. de Haan, R. E. Gur, C. Arango, C. M. Díaz-Caneja, C. H. Vinkers, and J. A. Vorstman, "Early interventions in risk groups for schizophrenia: what are we waiting for?," npj Schizophrenia, vol. 2, no. 1, pp. 1-9, 2016.

[5] E. Cheniaux, J. Landeira-Fernandez, and M. Versiani, "The Diagnoses of Schizophrenia, Schizoaffective Disorder, Bipolar Disorder and Unipolar Depression: Interrater Reliability and Congruence between DSM-IV and ICD-10," Psychopathology, vol. 42, no. 5, pp. 293-298, 2009.

[6] C. J. van Oel, M. M. Sitskoorn, M. P. Cremer, and R. S. Kahn, "School Performance as a Premorbid Marker for Schizophrenia: A Twin Study," Schizophrenia Bulletin, vol. 28, no. 3, pp. 401-414, 2002.

[7] J. Schiffman, H. J. Sorensen, J. Maeda, E. L. Mortensen, J. Victoroff, K. Hayashi, N. M. Michelsen, M. Ekstrom, and S. Mednick, "Childhood Motor Coordination and Adult Schizophrenia Spectrum Disorders," American Journal of Psychiatry, vol. 166, no. 9, pp. 1041-1047, 2009.

[8] A. H. Neuhaus, F. C. Popescu, J. Rentzsch, and J. Gallinat, "Critical Evaluation of Auditory Event-Related Potential Deficits in Schizophrenia: Evidence From Large-Scale Single-Subject Pattern Classification," Schizophrenia Bulletin, vol. 40, no. 5, pp. 1062-1071, 2013.

[9] M. Shim, H.-J. Hwang, D.-W. Kim, S.-H. Lee, and C.-H. Im, "Machine-learning-based diagnosis of schizophrenia using combined sensor-level and source-level EEG features," Schizophrenia Research, vol. 176, no. 2-3, pp. 314-319, 2016.

[10] A. H. Neuhaus, F. C. Popescu, J. A. Bates, T. E. Goldberg, and A. K. Malhotra, "Single-subject classification of schizophrenia 
using event-related potentials obtained during auditory and visual oddball paradigms," European Archives of Psychiatry and Clinical Neuroscience, vol. 263, no. 3, pp. 241-247, 2012.

[11] J. Laton, J. V. Schependom, J. Gielen, J. Decoster, T. Moons, J. D. Keyser, M. D. Hert, and G. Nagels, "Single-subject classification of schizophrenia patients based on a combination of oddball and mismatch evoked potential paradigms," Journal of the Neurological Sciences, vol. 347, no. 1-2, pp. 262-267, 2014

[12] S. Sur and V. Sinha, "Event-related potential: An overview," Industrial Psychiatry Journal, vol. 18, no. 1, p. 70, 2009.

[13] D. H. R. Blackwood and W. J. Muir, "Cognitive Brain Potentials and their Application," British Journal of Psychiatry, vol. 157, no. S9, pp. 96-101, 1990.

[14] I. Feinberg, "Efference Copy and Corollary Discharge: Implications for Thinking and Its Disorders," Schizophrenia Bulletin, vol. 4, no. 4, pp. 636-640, 1978.

[15] J. M. Ford, B. J. Roach, W. O. Faustman, and D. H. Mathalon, "Out-of-Synch and Out-of-Sorts: Dysfunction of Motor-Sensory Communication in Schizophrenia," Biological Psychiatry, vol. 63 , no. 8, pp. 736-743, 2008.

[16] K. Kilteni, B. J. Andersson, C. Houborg, and H. H. Ehrsson, "Motor imagery involves predicting the sensory consequences of the imagined movement," Nature Communications, vol. 9, no. 1, pp. 1-9, 2018.

[17] P. F. Sowman, A. Kuusik, and B. W. Johnson, "Self-initiation and temporal cueing of monaural tones reduce the auditory N1 and P2,' Experimental Brain Research, vol. 222, no. 1-2, pp. 149-157, 2012.

[18] J. M. Ford, V. A. Palzes, B. J. Roach, and D. H. Mathalon, "Did I Do That? Abnormal Predictive Processes in Schizophrenia When Button Pressing to Deliver a Tone," Schizophrenia Bulletin, vol. 40, no. 4, pp. 804-812, 2013.

[19] S. S. Shergill, G. Samson, P. M. Bays, C. D. Frith, and D. M. Wolpert, "Evidence for Sensory Prediction Deficits in Schizophrenia," American Journal of Psychiatry, vol. 162, no. 12, pp. 2384-2386, 2005.

[20] R. L. C. Mitchell, "Right hemisphere language functions and schizophrenia: the forgotten hemisphere?," Brain, vol. 128, no. 5 , pp. 963-978, 2005.

[21] K. Hugdahl, E.-M. Løberg, L. E. Falkenberg, E. Johnsen, K. Kompus, R. A. Kroken, M. Nygård, R. Westerhausen,

K. Alptekin, and M. Özgören, "Auditory verbal hallucinations in schizophrenia as aberrant lateralized speech perception: Evidence from dichotic listening," Schizophrenia Research, vol. 140, no. 1-3, pp. 59-64, 2012

[22] D. de Achával, M. F. Villarreal, E. Y. Costanzo, J. Douer, M. N. Castro, M. C. Mora, C. B. Nemeroff, E. Chu, K.-J. Bär, and S. M. Guinjoan, "Decreased activity in right-hemisphere structures involved in social cognition in siblings discordant for schizophrenia," Schizophrenia Research, vol. 134, no. 2-3, pp. 171-179, 2012

[23] Romanow, Cho, and Straub, "Riding the Wave: Past Trends and Future Directions for Health IT Research," MIS Quarterly, vol. 36, no. 3, pp. iii-x, 2012.

[24] I. Eigner and F. Bodendorf, "An intelligent decision support system for readmission prediction in healthcare," it - Information Technology, vol. 60, no. 4, pp. 195-205, 2018.

[25] I. Eigner, F. Bodendorf, and N. Wickramasinghe, "Predicting high-cost patients by Machine Learning: A case study in an Australian private hospital group," in Proceedings of 11th International Conference on Bioinformatics and Computational Biology, vol. 60, pp. 94-103, 2019.

[26] C. I. Ossai and N. Wickramasinghe, "Intelligent therapeutic decision support for 30 days readmission of diabetic patients with different comorbidities," Journal of Biomedical Informatics, vol. 107, p. 103486, 2020.

[27] Hevner, March, Park, and Ram, "Design Science in Information Systems Research," MIS Quarterly, vol. 28, no. 1, pp. 75-105, 2004.
[28] G. Deuschl and A. Eisen, Recommendations for the practice of clinical neurophysiology: guidelines of the International Federation of Clinical Neurophysiology. Elsevier, 1999.

[29] A. Bulling, J. A. Ward, H. Gellersen, and G. Tröster, "Eye Movement Analysis for Activity Recognition Using Electrooculography," IEEE Transactions on Pattern Analysis and Machine Intelligence, vol. 33, no. 4, pp. 741-753, 2011.

[30] F. Knolle, E. Schröger, and S. A. Kotz, "Prediction errors in self- and externally-generated deviants," Biological Psychology, vol. 92 , no. 2 , pp. 410-416, 2013.

[31] J. M. Ford, B. J. Roach, W. O. Faustman, and D. H. Mathalon, "Synch Before You Speak: Auditory Hallucinations in Schizophrenia," American Journal of Psychiatry, vol. 164, no. 3, pp. 458-466, 2007.

[32] L. Breiman, "Random Forests," Machine Learning, vol. 45, no. 1, pp. 5-32, 2001.

[33] A. Liaw and M. Wiener, "Classification and Regression by randomforest," $R$ News 2, vol. 2, no. 3, pp. 18-22, 2002.

[34] Y. Wang, Z. Wang, W. Clifford, C. Markham, T. E. Ward, and C. Deegan, "Validation of low-cost wireless EEG system for measuring event-related potentials," in IEEE ISSC 2018, pp. 1-6, 2018.

[35] D. C. Javitt and R. A. Sweet, "Auditory dysfunction in schizophrenia: integrating clinical and basic features," Nature Reviews Neuroscience, vol. 16, no. 9, pp. 535-550, 2015.

[36] R. van Lutterveld, A. Hillebrand, K. M. J. Diederen, K. Daalman, R. S. Kahn, C. J. Stam, and I. E. C. Sommer, "Oscillatory Cortical Network Involved in Auditory Verbal Hallucinations in Schizophrenia," PLoS ONE, vol. 7, no. 7, p. e41149, 2012.

[37] P. M. Llorca, B. Pereira, R. Jardri, I. Chereau-Boudet, G. Brousse, D. Misdrahi, G. Fénelon, A.-M. Tronche, R. Schwan, C. Lançon, A. Marques, M. Ulla, P. Derost, B. Debilly, F. Durif, and I. de Chazeron, "Hallucinations in schizophrenia and Parkinson's disease: an analysis of sensory modalities involved and the repercussion on patients," Scientific Reports, vol. 6, no. 1, pp. 1-9, 2016.

[38] C. Ogura, Y. Nageishi, M. Matsubayashi, F. Omura, A. Kishimoto, and M. Shimokochi, "Abnormalities in Event-Related Potentials, N100, P200, P300 and Slow Wave in Schizophrenia," Psychiatry and Clinical Neurosciences, vol. 45, no. 1, pp. 57-65, 1991.

[39] C. J. Simons, A. Sambeth, L. Krabbendam, S. Pfeifer, J. van Os, and W. J. Riedel, "Auditory P300 and N100 components as intermediate phenotypes for psychotic disorder: Familial liability and reliability," Clinical Neurophysiology, vol. 122, no. 10, pp. 1984-1990, 2011.

[40] W. J. Muir, D. M. S. Clair, and D. H. R. Blackwood, "Long-latency auditory event-related potentials in schizophrenia and in bipolar and unipolar affective disorder," Psychological Medicine, vol. 21, no. 4, pp. 867-879, 1991.

[41] S. P. Kutcher, "Auditory P300 in Borderline Personality Disorder and Schizophrenia," Archives of General Psychiatry, vol. 44, no. 7, pp. 645-650, 1987.

[42] H. K. Ising, F. Smit, W. Veling, J. Rietdijk, S. Dragt, R. M. C. Klaassen, N. S. P. Savelsberg, N. Boonstra, D. H. Nieman, D. H. Linszen, L. Wunderink, and M. van der Gaag, "Cost-effectiveness of preventing first-episode psychosis in ultra-high-risk subjects: multi-centre randomized controlled trial," Psychological Medicine, vol. 45, no. 7, pp. 1435-1446, 2014.

[43] R. Buettner, J. Frick, and T. Rieg, "High-performance detection of epilepsy in seizure-free EEG recordings: A novel machine learning approach using very specific epileptic EEG sub-bands," ICIS ' 19 Proc., pp. 1-16, 2019.

[44] R. Buettner, J. Fuhrmann, and L. Kolb, "Towards high-performance differentiation between Narcolepsy and Idiopathic Hypersomnia in 10 minute EEG recordings using a Novel Machine Learning Approach," in IEEE HealthCom 2019, pp. 1-6, 2019.

[45] R. Buettner, A. Grimmeisen, and A. Gotschlich, "High-performance diagnosis of sleep disorders: a novel, accurate and fast machine learning approach 
using electroencephalographic data," in HICSS'53 Proc., pp. 3246-3255, 2020.

[46] R. Buettner, T. Rieg, and J. Frick, "Machine Learning based Diagnosis of Diseases Using the Unfolded EEG Spectra: Towards an Intelligent Software Sensor," in Information Systems and Neuroscience, LNISO, vol. 32, pp. 165-172, Springer, 2020.

[47] H. Baumgartl, F. Dikici, D. Sauter, and R. Buettner, "Detecting Antisocial Personality Disorder Using a Novel Machine Learning Algorithm Based on Electroencephalographic Data.," in PACIS'20 Proc., 2020.

[48] H. Baumgartl, P. Roessler, D. Sauter, and R. Buettner, "Measuring Social Desirability Using a Novel Machine Learning Approach Based on EEG Data.," in PACIS'20 Proc., 2020.

[49] H. Baumgartl, S. Bayerlein, and R. Buettner, "Measuring extraversion using EEG data," Information Systems and Neuroscience, LNISO, vol. 43, pp. 259-265, 2020.

[50] T. Rieg, J. Frick, M. Hitzler, and R. Buettner, "High-performance detection of alcoholism by unfolding the amalgamated EEG spectra using the Random Forests method,' in HICSS'52 Proc., pp. 3769-3777, 2019.

[51] J. Gross, H. Baumgartl, and R. Buettner, "A Novel Machine Learning Approach for High-Performance Diagnosis of Premature Internet Addiction Using the Unfolded EEG Spectra," AMCIS'20 Proc., 2020.

[52] J. Breitenbach, H. Baumgartl, and R. Buettner, "Detection of Excessive Daytime Sleepiness in Resting-State EEG Recordings: A Novel Machine Learning Approach Using Specific EEG Sub-Bands and Channels," AMCIS'20 Proc, 2020.

[53] D. Raab, H. Baumgartl, and R. Buettner, "Machine Learning Based Diagnosis of Binge Eating Disorder Using EEG Recordings.,' in PACIS'20 Proc., 2020.

[54] T. Rieg, J. Frick, and R. Buettner, "Machine learning-based diagnosis of epilepsy in clinical routine: Lessons learned from a retrospective pilot study," Information Systems and Neuroscience, LNISO, vol. 32, pp. 165-172, 2020.

[55] R. Buettner, M. Buechele, B. Grimmeisen, and P. Ulrich, "Machine Learning Based Diagnostics of Developmental Coordination Disorder using Electroencephalographic Data," in HICSS'54 Proc., 2021, in press.

[56] R. Buettner, D. Beil, S. Scholtz, and A. Djemai, "Development of a machine learning based algorithm to accurately detect schizophrenia based on one-minute eeg recordings," in HICSS'53 Proc., pp. 3216-3225, 2020.

[57] R. Buettner, "Cognitive workload of humans using artificial intelligence systems: towards objective measurement applying eye-tracking technology," in $A A A I$ 2013, pp. 37-48, Springer, 2013.

[58] R. Buettner, "A User's Cognitive Workload Perspective in Negotiation Support Systems: An Eye-Tracking Experiment.," in PACIS'16 Proc., pp. 115-127, 2016.

[59] R. Buettner, "The relationship between visual website complexity, and a user's mental workload: A NeuroIS perspective," in Information Systems and Neuroscience, LNISO, vol. 16, pp. 107-113, Springer, 2017.

[60] R. Buettner, H. Baumgartl, and D. Sauter, "Microsaccades as a Predictor of a User's Level of Concentration," in Information Systems and Neuroscience, LNISO, vol. 29, pp. 173-177, Springer, 2019.

[61] S. Sauer, J. Lemke, W. Zinn, R. Buettner, and N. Kohls, "Mindful in a random forest: Assessing the validity of mindfulness items using random forests methods," Personality and Individual Differences, vol. 81, pp. 117-123, 2015.

[62] S. Sauer, R. Buettner, T. Heidenreich, J. Lemke, C. Berg, and C. Kurz, "Mindful Machine Learning," European Journal of Psychological Assessment, vol. 34, pp. 6-13, 2016.

[63] R. Buettner, S. Sauer, C. Maier, and A. Eckhardt, "Towards ex ante prediction of user performance: a novel NeuroIS methodology based on real-time measurement of mental effort," in HICSS'48 Proc., pp. 533-542, 2015.
[64] R. Buettner, L. Bachus, L. Konzmann, and S. Prohaska, "Asking Both the User's Heart and Its Owner: Empirical Evidence for Substance Dualism," in Information Systems and Neuroscience, LNISO, vol. 29, pp. 251-257, Springer, 2019.

[65] A. Eckhardt, C. Maier, J. Hsieh, T. Chuk, A. B. Chan, J. H. Hsiao, and R. Buettner, "Objective Measures of IS Usage Behavior Under Conditions of Experience and Pressure Using Eye Fixation Data,' ICIS '13 Proc., pp. 1-14, 2013.

[66] R. Buettner and M. Schunter, "Efficient machine learning based detection of heart disease," in IEEE HealthCom 2019, pp. 1-6, 2019.

[67] R. Buettner, "Robust User Identification Based on Facial Action Units Unaffected by Users' Emotions," in HICSS'51 Proc., pp. 265-273, 2018.

[68] R. Buettner, S. Sauer, C. Maier, and A. Eckhardt, "Real-time prediction of user performance based on pupillary assessment via eye tracking," AIS Transactions on Human-Computer Interaction, vol. 10, no. 1, pp. 26-56, 2018.

[69] R. Buettner, "Towards a new personal information Technology Acceptance Model: Conceptualization and empirical evidence from a bring your own device dataset," AMCIS '15 Proc., pp. 1-14, 2015.

[70] R. Buettner, "Analyzing the Problem of Employee Internal Social Network Site Avoidance: Are Users Resistant due to their Privacy Concerns?," in HICSS'48 Proc., pp. 1819-1828, 2015.

[71] R. Buettner, "Predicting user behavior in electronic markets based on personality-mining in large online social networks," Electronic Markets, vol. 27, no. 3, pp. 247-265, 2017.

[72] R. Buettner, "Getting a job via career-oriented social networking markets," Electronic Markets, vol. 27, no. 4, pp. 371-385, 2017.

[73] R. Buettner, "A Systematic Literature Review of Crowdsourcing Research from a Human Resource Management Perspective," in HICSS'48 Proc., pp. 4609-4618, 2015.

[74] J. Landes and R. Buettner, "Job allocation in a temporary employment agency via multi-dimensional price VCG auctions using a multi-agent system," in MICAI 2011, pp. 182-187, 2011.

[75] R. Buettner and J. Landes, "Web Service-based Applications for Electronic Labor Markets: A Multi-dimensional Price VCG Auction with Individual Utilities," in ICIW 2012, pp. 168-177, 2012.

[76] S. C. Rodermund, R. Buettner, and I. J. Timm, "Towards Simulation-based Preplanning for Experimental Analysis of Nudging,"WI-2020 Proc., vol. 12, pp. 14-29, 2020.

[77] R. Buettner, "The Impact of Trust in Consumer Protection on Internet Shopping Behavior: An Empirical Study using a Large Official Dataset from the European Union," in IEEE BigDataService 2020, pp. 66-69, 2020.

[78] H. Baumgartl and R. Buettner, "Development of a highly precise place recognition module for effective human-robot interactions in changing lighting and viewpoint conditions," HICSS'53 Proc., pp. 563-572, 2020.

[79] H. Baumgartl, J. Tomas, R. Buettner, and M. Merkel, "A deep learning-based model for defect detection in laser-powder bed fusion using in-situ thermographic monitoring," Progress in Additive Manufacturing, vol. 5, pp. 277-285, 2020.

[80] R. Buettner and H. Baumgartl, "A highly effective deep learning based escape route recognition module for autonomous robots in crisis and emergency situations," in HICSS' 52 Proc., pp. 659-666, 2019.

[81] H. Baumgartl, J. Tomas, R. Buettner, and M. Merkel, "A novel Deep-Learning Approach for Automated Non-Destructive Testing in Quality Assurance based on Convolutional Neural Networks," ACEX-2019 Proc., 2019.

[82] J. Gross, J. Breitenbach, H. Baumgartl, and R. Buettner, "High Performance Detection of Corneal Ulceration Using Image Classification with Convolutional Neural Networks," in HICSS' 54 Proc., 2021, in press. 